\title{
Hypersalivation in a Patient with Anti-NMDAR Encephalitis with Ovarian Teratoma
}

\author{
Yoshiharu Taguchi, Shutaro Takashima, Takamasa Nukui and Kortaro Tanaka \\ Key words: anti-NMDAR encephalitis, hypersalivation, ovarian teratoma, orofacial dyskinesia
}

(Inter Med 49: 803-804, 2010)

(DOI: 10.2169/internalmedicine.49.3221)
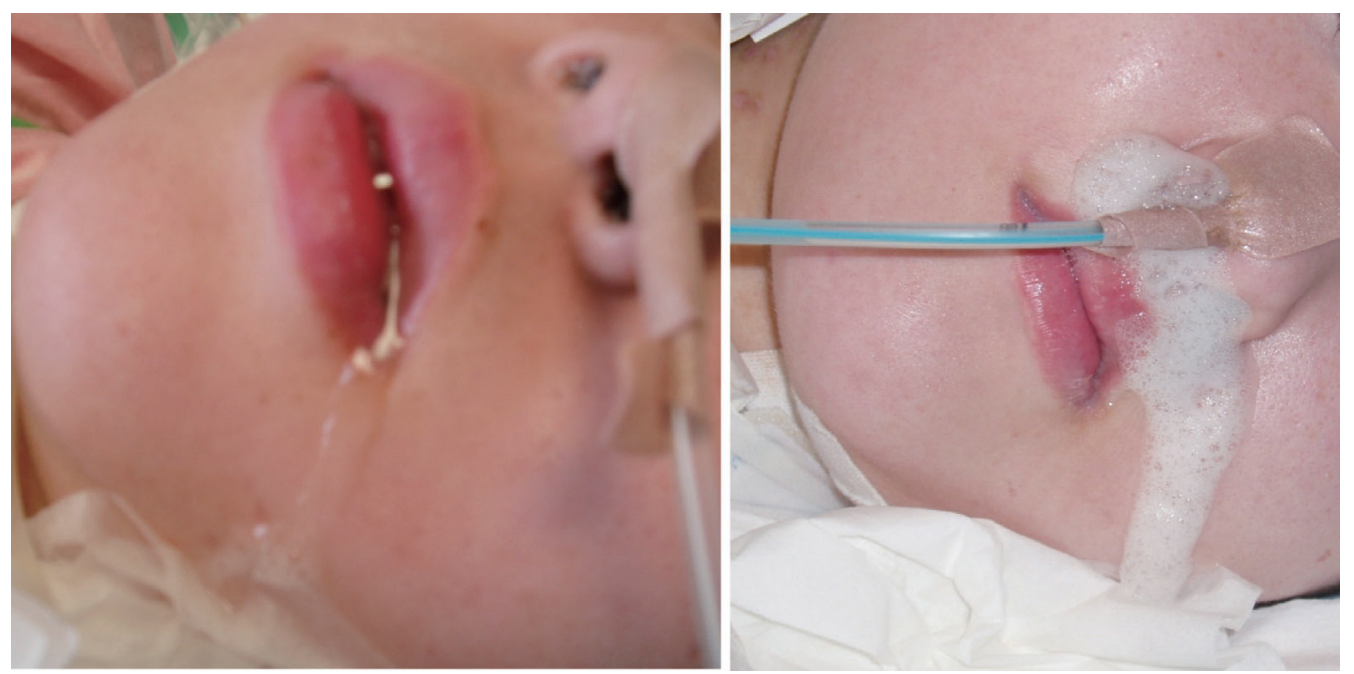

Picture. A very large volume of saliva flowed from her mouth and nose resembling "bubbles of a crab".

A 17-year-old woman, who had suffered from orbital pain and sleep disturbance for a few days, was admitted to our hospital with progressive psychosis, and emotional and behavioural changes. On admission, she presented with a confusional state and pyrexia $\left(38.1^{\circ} \mathrm{C}\right)$. Physical examination revealed orofacial dyskinesia such as chewing, hypersalivation and neck stiffness. Her symptoms gradually worsened, and on the forth hospital day mechanical ventilatory support was required for her convulsive seizures and severe hypersalivation. Her diffusion-weighted image revealed a hyperintense lesion in the left hippocampus. A pelvic CT demonstrated a teratoma in the left ovary. On the 42 nd day, she underwent unilateral salpingo-oophorectomy. The histopathological diagnosis was mature ovarian teratoma. Antibodies to NR1/ NR2B heteromers of NMDAR (N-methyl-D-aspartate receptor) in serum and CSF were positive. Then, she was diagnosed as having anti-NMDAR encephalitis with an ovarian teratoma. Although she received anticonvulsants, methylprednisolone pulse therapy and intravenous immunoglobulin therapy, her symptoms were not completely recovered. When her anesthetic level which was controlled by thiopental sodium and midazolam was decreased, her orofacial dyskinesia and hypersalivation immediately worsened. She needed continuous mechanical aspiration support due to the large volume of saliva which flowed from her mouth and nose, resembling "bubbles of a crab" (Picture). In contrast, when her anesthetic level was increased, her orofacial dyskinesia and hypersalivation disappeared. Her hypersalivation continued for 7 months.

It has been reported that patients with anti-NMDAR encephalitis often have accompanying orofacial dyskinesia and hypersalivation $(1,2)$. Thus, clinical practitioners should suspect the diagnosis of anti-NMDAR encephalitis, when a patient exhibits orofacial dyskinesia and hypersalivation like 
"bubbles of a crab".

\section{References}

1. Kamei S, Kuzuhara S, Ishihara M, et al. Nationwide survey of acute juvenile female non-herpetic encephalitis in Japan: relationship to anti-N-methyl-D-aspartate receptor encephalitis. Intern Med 48: 673-679, 2009.
2. Dalmau J, Gleichman AJ, Hughes EG, et al. Anti-NMDA-receptor encephalitis: case series and analysis of the effects of antibodies. Lancet Neurol 7: 1091-1098, 2008.

(C) 2010 The Japanese Society of Internal Medicine http://www.naika.or.jp/imindex.html 